Volumen 16 Número 3, Julio - Septiembre 2021, pp. 1-20, e605

THE ECONOMICS AND FINANCE EFFECTS OF THE COVID-19 PANDEMIC Editor: Dr. Ignacio Perrotini

DOI: https://doi.org/10.21919/remef.v16i3.605

(Recibido: 25/febrero/2021, aceptado: 28/mayo/2021, publicado: 10/junio/2021)

\title{
Volatilidad y COVID-19: evidencia empírica internacional
}

\author{
Tomás Gómez Rodríguez ${ }^{1}$ - Universidad Autónoma del Estado de Hidalgo, México \\ Humberto Ríos Bolívar - Instituto Politécnico Nacional, México \\ Adriana Zambrano Reyes - Universidad Autónoma del Estado de Hidalgo, México
}

Se estudió la relación entre los efectos de la pandemia de COVID-19 y la volatilidad realizada. Además, se analizó el posible nexo entre las medidas de mitigación y contención adoptadas por los gobiernos y la volatilidad realizada. El período de estudio abarca del 2 de febrero de 2020 al 28 de agosto del mismo año. Este período a su vez se dividió en catorce muestras dos para cada mes, lo que dio origen a los catorce paneles no balanceados usados en las estimaciones. El método de estimación utilizado es Mínimos Cuadrados Ordinarios con efectos fijos de dos vías y el método de Mínimos Cuadrados Generalizados Estimados (EGLS) con efectos aleatorios de una vía (período). Se utilizaron datos de volatilidad realizada de sesenta y cinco de los principales índices bursátiles. Los resultados exhiben evidencia que apoya la existencia de una relación estadística significativa entre las medidas de contención y mitigación y la volatilidad realizada, principalmente en los meses de febrero y marzo, sin embargo, no se encontró uniformidad en los resultados. Por otro lado, no se encontró evidencia suficiente a favor de la existencia de una relación positiva entre los efectos de la pandemia COVID-19 y la volatilidad realizada.

Clasificación JEL: C33, G10, G15, G18, H12.

Palabras clave: Sistema Financiero, panel de datos, volatilidad en los mercados accionarios, COVID-19, medidas de contención y mitigación.

\section{Volatility and COVID-19: international empirical evidence}

The relationship between the effects of the COVID-19 pandemic and realized volatility was studied. In addition, the possible link between mitigation and containment measures adopted by governments and realized volatility was analyzed. The study period covers February 2, 2020 to August 28, 2020. This period in turn was divided into fourteen samples, two for each month, which gave rise to the fourteen unbalanced panels used in the estimations. The estimation method used is Ordinary Least Squares with two-way fixed effects and the Estimated Generalized Least Squares (EGLS) method with one-way random effects (period). Realized volatility data from sixty-five major stock market indexes were used. The results show evidence that supports the existence of a significant statistical relationship between containment and mitigation measures and realized volatility, mainly in the months of February and March; however, no uniformity was found in the results. On the other hand, not enough evidence was found in favor of the existence of a positive relationship between the effects of the COVID-19 pandemic and realized volatility.

JEL Classification: C33, G10, G15, G18, H12.

Keywords: Financial System, panel data, stock market volatility, COVID-19, containment and mitigation measures.

${ }^{1}$ Autor de correspondencia. Dirección: Carretera Apan-Calpulalpan Km.8, Col. Chimalpa, C.P. 43920 Apan, Hgo. Teléfono: 017717172000 extensión 5806. Correo electrónico: tomas_gomez@uaeh.edu.mx.

*Sin fuente de financiamiento para el desarrollo de la investigación 


\section{Introducción}

El coronavirus (COVID-19) es una enfermedad infecciosa causada por el nuevo tipo de coronavirus SARS-CoV-2 se originó en la ciudad china de Wuhan. Desde ese momento esta enfermedad ha estado causando estragos alrededor del mundo convirtiéndose rápidamente en una crisis sanitaria global (Ashraf, 2020a). El 11 de marzo de 2020 la Organización Mundial de la Salud declaró oficialmente que el brote de COVID-19 se había convertido en una pandemia mundial (Zhang, Hu y Ji, 2020).

El brote de la enfermedad altamente contagiosa COVID-19 fue un evento sorpresivo que provoco una incertidumbre sin precedentes con respecto a que tan alta es la mortalidad de este padecimiento y si es posible obtener una vacuna y cuándo (Ashraf, 2020b). Debido a estos los inversionistas y los mercados se enfrentan a un alto grado de incertidumbre con respecto a los impactos tanto económicos como financieros del virus (Baek, Mohanty, y Glambosky, 2020).

En respuesta al brote los gobiernos de todo el mundo se apresuraron a adoptar acciones de emergencia, como cierres forzosos, restricciones de viaje, pruebas y cuarentenas, además de paquetes económicos. El principal objetivo de estas acciones era asegurar el distanciamiento social entre las personas para contener la propagación de la enfermedad, por un lado y por el otro, minimizar el impacto económico adverso. Sin embargo, estas acciones generaron una incertidumbre adicional con respecto a su eficacia y sus repercusiones. Por ejemplo, los cierres si bien podían ser eficaces para reducir el número de casos, también podrían tener efectos negativos sobre el desempeño económico perjudicando así los empleos e ingresos de decenas de millones de personas (Ashraf, 2020b). Así mismo, el efecto adverso de la pandemia puede ser especialmente grave en algunas industrias como el turismo y la aviación (Zhang, Hu y Ji, 2020).

Como ya se ha mencionado la pandemia de COVID-19 ha desencadenado una serie de intervenciones gubernamentales sin precedentes. A fines de marzo de 2020, más de 100 países de todo el mundo ya habían instituido cierres parciales o totales y los viajes aéreos e interurbanos se habían reducido en un 70\% a 90\% en comparación con las cifras de marzo de 2019 en las principales ciudades del mundo, lo que afectó a miles de millones de personas (Dunford et al., 2020). Por otra parte, los gobiernos desde los ministerios de finanzas hasta los bancos centrales están desplegando paquetes de apoyo y estímulo para contener los daños económicos (Ashraf, 2020a).

Aunque todavía no están claras las repercusiones económicas mundiales exactas los mercados financieros ya han respondido con movimientos drásticos. En marzo de 2020, el mercado de valores de los Estados Unidos activo el mecanismo de cortafuegos cuatro veces en diez días. Desde su creación en 1987, el interruptor sólo se había activado una vez en 1997. Junto con la caída de los mercados bursátiles en Estados Unidos, los mercados bursátiles de Europa y Asia también se han hundido. El índice Financial Times Stock Exchange (FTSE) el cual es el principal índice del Reino Unido cayó más del 10\% el 12 de marzo de 2020, en su peor día desde 1987. El índice de referencia del mercado de valores de Japón (Nikkei) se desplomó más del 20\% desde su posición más alta en diciembre de 2019. Los bancos centrales y las autoridades respondieron inmediatamente lanzando sus instrumentos de política al mercado. Por ejemplo, el 15 de marzo de 2020 la Reserva Federal (FED) anunció una política de tasa de interés del cero por ciento y un programa de alivio cuantitativo (QE) de al menos 700,000 millones de dólares. Tras las respuestas negativas a esta política en el mercado, la FED anunció una política de QE ilimitada ocho días después (Zhang, Hu y Ji, 2020). 
La volatilidad es primordial para el funcionamiento de los mercados financieros. Actúa como un barómetro del riesgo financiero, el estrés o la incertidumbre que rodea a las inversiones financiera. Por lo tanto, es de una importancia crítica para los administradores de fondos, los inversores minoristas, así como para los directores financieros de las empresas. La literatura financiera ha establecido desde hace mucho tiempo un vínculo entre las crisis, las intervenciones gubernamentales, la incertidumbre política y la volatilidad de los mercados financieros (Zaremba, Kizys, Aharon y Demir, 2020)

Así mismo, el papel fundamental de la volatilidad se divide en dos, primero como medida del movimiento de los precios y segundo en su rol en la medición de la incertidumbre en los mercados y los precios. Si bien, el nivel de referencia en los mercados financieros como el S\&P 500 se interpreta como el precio del mercado, entonces la volatilidad del S\&P 500 puede interpretarse como la confianza que los participantes del mercado tienen en ese precio. Si los participantes en el mercado no están seguros del futuro o si son propensos a alterar su visión rápidamente y de forma completa, por ejemplo, a la luz de los informes de ganancias emergentes, de los anuncios de los bancos centrales o de la ocurrencia de un desastre natural entonces se podría esperar que las grandes oscilaciones de precios sean comunes. Si, por otra parte, en el mercado los participantes confían en que los precios actuales se aproximan a su supuesto valor justo, y no cambian de opinión a corto plazo, entonces se podría esperar sólo pequeñas fluctuaciones en los precios de un día para otro, sin que haya un cambio material en las circunstancias. Por tanto, el grado de estabilidad o fluctuación de los precios es indicativo del nivel de confianza compartido por los participantes en el mercado. Esto explica en parte por qué la volatilidad se incorpora frecuentemente como la principal medida de riesgo (Edwards y Lazzara, 2016). El reciente brote de COVID-19 ha dado lugar a una volatilidad sin precedentes en los mercados financieros de los Estados Unidos. Por ejemplo, el Índice de Volatilidad del CBOE (VIX) superó los 80 puntos el 16 de marzo de 2020, superando su récord de 2008 (Baek, Mohanty, y Glambosky, 2020).

Hasta el momento existen pocos trabajos que subrayan el impacto de COVID-19 en la volatilidad financiera algunos ejemplos son: Ali, Alam y Rizvi (2020), Baker et al. (2020), Albulescu (2020a), Albulescu (2020b), Haroon y Rizvi (2020), Zhang, Hu y Ji (2020), Onali (2020), Baek, Mohanty, y Glambosky (2020) y Zaremba, Kizys, Aharon y Demir (2020)

Este estudio contribuye a la reciente literatura sobre COVID-19 y volatilidad de tres maneras distintas: En primer lugar, el período utilizado es considerablemente mayor que en otros estudios, además este período se divide en catorce muestras lo que permite ser más específico en cuanto a en que momento fue mayor el efecto de las variables consideradas. En segundo lugar, este estudio complementa la literatura que ha surgido recientemente al utilizar tres índices de respuesta gubernamental a la pandemia de COVID-19. Por último, se emplea una medida de volatilidad realizada diferente a la utilizada en otros trabajos.

En este estudio se analiza la relación entre las consecuencias de COVID-19 y la volatilidad en los mercados financieros. Así mismo se investiga el efecto de las medias de mitigación y contención del brote de COVID-19 sobre la volatilidad en los mercados financieros. Particularmente se consideran tres hipótesis. Por un lado, se espera que las medidas gubernamentales estrictas de distanciamiento social, analizadas por el índice de rigor tengan un efecto positivo sobre la volatilidad en los índices bursátiles. Por otro lado, los anuncios de las políticas de contención y de atención sanitaria del gobierno (índice de contención sanitaria) conducen a una disminución de la volatilidad 
en el mercado de valores. Y, por último, la respuesta del gobierno al brote de COVID-19 lleva a una disminución de la volatilidad en los índices bursátiles. En cuanto a la relación entre las consecuencias de COVID-19 y la volatilidad realizada se asume que tanto el crecimiento de casos confirmados, como el número de decesos confirmados tienen un efecto positivo sobre la volatilidad

Para realizar el análisis empírico se analiza un período de tiempo que abarca del 2 de febrero al 28 de agosto de 2020. Este período de tiempo a su vez es dividido en catorce muestras es decir dos muestras para cada mes y se consideran sólo los días en que los mercados financieros operan. La variable independiente es la volatilidad realizada está es calculada para los principales índices accionarios de 65 países. Conforme a la fórmula propuesta por Chun, Cho y Ryu (2020). Para estudiar los efectos de COVID-19 se utiliza el número de casos y decesos confirmados. Mientras que para examinar las medidas de mitigación y contención de la pandemia de COVID-19 utilizamos el índice de restricción, el índice de respuesta gubernamental y el índice de contención sanitaria. Se utiliza el método de Mínimos Cuadrados Ordinarios con efectos fijos de dos vías y el método de Mínimos Cuadrados Generalizados Estimados (EGLS) con efectos aleatorios de una vía (período) para realizar las estimaciones.

El resto del trabajo se divide en las siguientes secciones: en la sección revisión de la literatura se muestran los trabajos asociados a la relación entre las medidas de contención y la volatilidad, así como, la literatura relacionada a las consecuencias del COVID-19 y la volatilidad. En la sección datos se encuentran la descripción de las variables y sus estadísticas básicas; en la sección metodología se describen los métodos de estimación y los modelos a estimar. En la sección resultados, se presentan los hallazgos y análisis del modelo estimado. Por último, en las conclusiones se consignan las principales aportaciones del estudio.

\section{Revisión de la literatura}

El surgimiento de la pandemia de COVID-19 ha provocado la aparición de nuevas ramas de la literatura en el ámbito económico entre las cuales se destacan tres: la primera relaciona la incertidumbre económica con las consecuencias originadas por la pandemia, la segunda rama estudia el nexo entre los efectos de la incertidumbre provocada por la pandemia de COVID-19 y los mercados financieros, por último, la tercera rama se ocupa de los efectos del COVID-19 sobre la volatilidad en los mercados financieros.

Algunos de los trabajos que estudian la relación entre la incertidumbre creada por la pandemia y la economía son: Ludvigson, Ma, y Ng (2020), Baker Bloom y Terry (2020), Dietrich, Kuester, Müller y Schoenle (2020), Norouzi, Zarazua de Rubens, Choupanpiesheh y Enevoldsen (2020), Donthu y Gustafsson (2020). En el trabajo de Ludvigson, Ma, y Ng (2020) se intenta cuantificar el impacto que tienen los desastres naturales sobre la macroeconomía y además incorpora estos resultados al análisis del probable impacto de COVID-19 mediante la construcción de una serie con datos mensuales que considera los desastres que han ocurrido durante el período 1980-2019. Específicamente, analizan el impacto del COVID-19 sobre la actividad económica y la incertidumbre empleando el modelo de vectores autorregresivos (VAR); sus hallazgos indican que en el caso de una duración de tres meses de la pandemia ocasionada por COVID-19 se observaría una caída acumulada de doce por ciento en la actividad industrial y la existencia de por lo menos cinco 
meses de incertidumbre macroeconómica.

Baker, Bloom y Terry (2020) se preguntan si existe una relación causal entre el ciclo económico y la incertidumbre. Para estudiar esta relación construyen un panel de datos de varios países de los niveles del mercado accionario y la volatilidad, además como instrumentos utilizan datos acerca de desastres naturales, ataques terroristas y perturbaciones políticas en estimaciones VAR. Estiman que el COVID-19 reducirá el PIB de los Estados Unidos en 9\% para este año. Por último, en el trabajo de Dietrich, Kuester, Müller y Schoenle (2020) se realizó una encuesta en los hogares acerca de qué expectativas tienen sobre las consecuencias económicas de la pandemia provocada por el COVID-19. Esta encuesta tiene una frecuencia diaria y es en tiempo real. Los datos obtenidos en las encuestas se introducen en un modelo de ciclo de negocios Nuevo Keynesiano. Los resultados del modelo muestran que el impacto económico de la pandemia en el corto plazo depende principalmente de la política monetaria.

Otro estudio relacionado con el efecto de la incertidumbre causada por el COVID-19 sobre la demanda del consumidor, es el trabajo de Norouzi, Zarazua de Rubens, Choupanpiesheh y Enevoldsen (2020), los autores desarrollan un modelo comparativo de redes neuronales para analizar los impactos del COVID-19 en la demanda de electricidad y petróleo en China. El análisis indica que la severidad de la epidemia afecta de manera significativa la demanda de electricidad y petróleo, tanto directa como indirectamente. Los resultados mencionados muestran que el nivel de la pandemia tiene un impacto significativo sobre la demanda de energía y sobre las decisiones de consumo de la población.

Por último, la pandemia de COVID-19 no sólo afecta la salud de las personas, se trata de una crisis social que está afectando todos los aspectos de la vida cotidiana. En respuesta al brote pandémico los líderes de muchos países decidieron salvar vidas antes de salvar la economía, declarando cierres repentinos o escalonados en sus países. Como parte de la estrategia de salvar vidas humanas, se implementaron políticas como distanciamiento social y quedarse en casa, estrategias que cambiaron radicalmente las expectativas de las personas sobre sus decisiones de consumo, estas estrategias dañaron gravemente a varias ramas del comercio y de la industria productiva, como se señala en el trabajo de Donthu y Gustafsson (2020).

Por otro lado, se encuentra la literatura que examina el impacto del COVID-19 en los resultados del sector financiero. En este sentido, el trabajo de Schell Wang y Huynh (2020) estudia las diferencias en las reacciones de los mercados bursátiles a noticias relacionadas con enfermedades, analizando los rendimientos anormales de las acciones mundiales. Encontrando que sólo el COVID-19 tuvo un efecto negativo significativo en los mercados bursátiles el cual duró por lo menos 30 días.

Por otra parte, Corbet, Hou, Hu, Lucey y Oxley (2020a) examinan cual es el efecto sobre la rentabilidad de las acciones si la palabra corona aparece en el nombre de la empresa, ellos descubren que las empresas con la palabra corona en sus nombres experimentaron fuertes rendimientos negativos y un aumento excepcional de la volatilidad cuando se anunció la pandemia de COVID-19. De igual modo, Sharif, Aloui y Yarovaya (2020) encuentran que para el caso de Estados Unidos la pandemia afecta en mayor medida al riesgo geopolítico y a la incertidumbre económica mientras que el mercado bursátil no se ve afectado en la misma medida. Alfaro, Chari, Greenland y Schott (2020) utilizan datos de los Estados Unidos y constatan que el valor del mercado de valores disminuyó en respuesta a pandemias como la de COVID-19 y el síndrome respiratorio agudo grave (SRAS). Así 
mismo, Al-Awadhi, Alsaifi, Al-Awadhi y Alhammadi (2020) constataron que los precios generales de las acciones disminuyeron en China debido a los resultados económicos adversos previstos por el COVID-19. Ashraf (2020a) examinó los datos de 64 países y descubrió que los mercados bursátiles en general reaccionaron negativamente al brote de COVID-19. Sin embargo, esta reacción sólo fue significativa para el aumento del número de casos confirmados, pero no para el aumento del número de decesos. Por otro lado, las estrictas medidas de distanciamiento social han debilitado de manera significativa la reacción negativa de los mercados bursátiles al crecimiento de los casos confirmados de COVID-19. En este sentido, el estudio de Ashraf (2020b) encuentra que el hecho de evitar una mayor incertidumbre a nivel nacional refuerza significativamente la reacción negativa de los mercados bursátiles al crecimiento de los casos confirmados de COVID-19.

Analizando si diferentes activos pueden tener propiedades que los clasifiquen como un paraíso seguro, Corbet, Larkin y Lucey (2020b), Conlon y McGee (2020) y Conlon, Corbet y McGee (2020) concluyen que los activos criptográficos en gran medida no actúan como coberturas, o refugios seguros, sino más bien como amplificadores del contagio durante el mercado bajista en medio de la pandemia. Por otro lado, Goodell y Goutte (2020) analizan la reacción del Bitcoin a las muertes diarias en el mundo por COVID-19 y muestran que el Bitcoin es un activo de refugio seguro. Además, Sharif, Aloui y Yarovaya (2020) encuentran que los futuros del oro y la soya tuvieron un fuerte papel de refugio durante el brote de COVID-19.

Por último, se encuentran los estudios que examinan el impacto de COVID-19 sobre la volatilidad en los mercados financieros. Algunos trabajos que examinan esta área son: Ali, Alam y Rizvi (2020), Albuquerque, Koskinen, Yang y Zhang (2020), Baker et al. (2020), Albulescu, (2020a), Albulescu (2020b) Haroon y Rizvi (2020), Zhang, Hu y Ji (2020), Onali (2020), Baek Mohanty y Glambosky (2020) y Zaremba, Kizys, Aharon y Demir (2020). Un ejemplo de la investigación en esta área es el trabajo de Baker et al. (2020) el cual compara la reacción del mercado de valores de los Estados Unidos a varias enfermedades infecciosas. Ellos detectan que, de todas las enfermedades consideradas en el estudio, solo el COVID-19 ha generado un aumento significativo de la volatilidad en los Estados Unidos. Así mismo, en el trabajo de Ali, Alam y Rizvi (2020) se analiza la reacción de los mercados financieros globales en términos del rendimiento y de la volatilidad, conforme el epicentro de la pandemia se desplazó de China hacia Europa y luego hacia los Estados Unidos. Sus hallazgos sugieren que mientras en China los mercados se han estabilizado, los mercados globales han experimentado una caída importante especialmente en la última fase de la propagación.

Para investigar empíricamente el efecto que tienen los anuncios oficiales acerca del COVID19 sobre la volatilidad de los mercados financieros en los Estados Unidos Albulescu (2020b), considera tanto datos de Estados Unidos como a nivel global y concluye que la crisis sanitaria aumenta la volatilidad realizada en el índice S\&P 500. Así mismo, Albulescu (2020a) analiza el efecto de los anuncios oficiales de nuevos casos de infección y el índice de mortalidad sobre el índice de volatilidad de los mercados financieros VIX. Mientras que los nuevos casos notificados en China y fuera de China tienen un efecto mixto sobre la volatilidad financiera, el índice de mortalidad influye positivamente en el VIX. Además, en este estudio se encuentra que cuanto mayor es el número de países afectados, mayor es la volatilidad financiera. De igual manera, Zhang, Hu y Ji (2020) examinaron la volatilidad en diez mercados bursátiles en los países con más casos confirmados durante los meses de enero y febrero de 2020, y encontraron que la volatilidad aumentó 
sustancialmente en febrero debido al COVID-19. Centrándose en los rendimientos de los mercados de valores Zaremba, Kizys, Aharon y Demir (2020) examinan si la respuesta del gobierno al COVID19 mitiga la volatilidad de los mercados bursátiles internacionales. Ellos documentan un aumento significativo de la volatilidad de los mercados de valores en los países en los que los gobiernos adoptan medidas rigurosas para frenar la propagación de COVID-19, como campañas de información y cancelación de eventos públicos.

Por otro lado, en el trabajo Onali (2020) se identificó que aumentos significativos en la volatilidad de los mercados bursátiles de los Estados Unidos están relacionados a los informes de casos de COVID-19 y muertes en múltiples países. En el marco de la pandemia de COVID-19 existe la posibilidad de que haya variaciones en la volatilidad entre las distintas industrias, por ejemplo, las empresas de mayor calificación ambiental y social exhiben una menor volatilidad en el rendimiento de las acciones de acuerdo al trabajo de Albuquerque, Koskinen, Yang y Zhang (2020). Por otro lado, Haroon y Rizvi (2020) investigan si la cobertura de noticias sobre COVID-19 provoca cambios en la volatilidad. Sus resultados muestran que para los distintos sectores económicos las noticias cargadas de pánico contribuyen en mayor medida a un aumento de la volatilidad en los sectores que se consideran más afectados por el brote de coronavirus. Utilizando los métodos de selección de características a través del aprendizaje automático Baek, Mohanty, y Glambosky (2020), eligen los indicadores económicos que mejor explican los cambios en la volatilidad. Sus resultados muestran que la volatilidad es sensible a las noticias acerca de COVID-19, Además, encuentran que tanto las noticias buenas como las malas son significativas, sin embargo, las noticias malas tienen mayor impacto sobre la volatilidad.

Por tanto, podemos clasificar los trabajos antes mencionados en dos ramas la primera rama se enfoca en el análisis del impacto de las noticias o anuncio gubernamentales relacionados al COVID19 sobre la volatilidad de los mercados accionarios (Albulescu 2020a, Albulescu 2020b, Onali 2020, Haroon y Rizvi 2020, Baek, Mohanty y Glambosky 2020 y Haroon y Rizvi 2020). En la segunda rama están los estudios que examinan el efecto del número de casos y decesos sobre el rendimiento de las acciones el cual se utiliza como medida de volatilidad (Ali, Alam y Rizvi 2020, Zaremba, Kizys, Aharon y Demir 2020, Albuquerque, Koskinen, Yang y Zhang 2020). La presente investigación se centra en la segunda rama y se enfoca exclusivamente en una media de volatilidad realizada la cual fue propuesta por Chun, Cho y Ryu (2020) y se da un enfoque diferente al utilizar catorce muestras en lugar de una, resultado de segmentar el período de tiempo que se consideró para el estudio.

\section{Datos}

Para realizar el análisis empírico propuesto por este estudio, se recogieron datos de dos fuentes principales: la primera fuente fue www.investing.com y la segunda es la base de datos Oxford COVID19 Government Response Tracker (OxCGRT). Los datos sobre el nivel máximo y el nivel mínimo para cada día de los principales índices de las bolsas de valores más importantes se recogieron en el sitio web www.investing.com. Se obtuvieron datos para 65 países. Para mantener la coherencia se elige un solo índice bursátil para cada país de la muestra. En la tabla 1 se muestra la lista de países. Para construir la medida de volatilidad realizada, es importante calcular correctamente la duración de los movimientos de los precios dentro de un determinado período. En este estudio, adoptamos el método 
mencionado en el trabajo de Chun, Cho y Ryu (2020) como se muestra en la ecuación (1). A pesar de su simplicidad, este método suele estimar con éxito la volatilidad diaria del mercado

$$
r v_{i t}=\frac{P H_{i t}-P L_{i t}}{\left(P H_{i t}+P L_{i t}\right) / 2}
$$

Donde $r v_{i t}$ es la volatilidad realizada diaria para cada índice por país, $P H_{i t}$ es el nivel máximo del índice diario para cada índice por país, $P L_{i t}$ es el nivel mínimo diario del índice por país.

Tabla 1. Lista de países e índices bursátiles.

\begin{tabular}{|c|c|c|c|}
\hline País & Índice bursátil & País & Índice bursátil \\
\hline Argentina & S\&P Merval & Mongolia & MNE Top 20 \\
\hline Australia & S\&P ASX 200 & Marruecos & Moroccan All Shares \\
\hline Austria & ATX & Namibia & NSX Overall \\
\hline Bahrain & Bahrain All Share & Países Bajos & $\mathrm{AEX}$ \\
\hline Bangladesh & DSE 30 & Nueva Zelanda & NZX 50 \\
\hline Bélgica & BEL 20 & Nigeria & NSE 30 \\
\hline Brasil & Bovespa & Noruega & OSE Benchmark \\
\hline Bulgaria & SOFIX & Oman & MSM 30 \\
\hline Canada & S\&P TSX & Pakistan & Karachi 100 \\
\hline Chile & S\&P CLX IPSA & Peru & S\&P Lima General \\
\hline China & Shanghai & Filipinas & PSEi Composite \\
\hline Colombia & COLCAP & Polonia & WIG 30 \\
\hline República Checa & MSCI Czech Republic & Portugal & PSI 20 \\
\hline Dinamarca & OMX Copenhagen 20 & Qatar & QE General \\
\hline Egipto & EGX 70 EWI & Rumania & BET \\
\hline Finlandia & MSCI Finland & Rusia & MOEX \\
\hline Francia & CAC 40 & Arabia Saudita & Tadawul All Share \\
\hline Alemania & DAX & Serbia & BELEX 15 \\
\hline Grecia & Athens General & Singapur & MSCI Singapore \\
\hline Hong Kong & Dow Jones Hong Kong & Eslovaquia & SAX \\
\hline Hungría & BSE & Eslovenia & Blue-Chip SBITOP \\
\hline Islandia & ICEX Main & Sudafrica & TOP 40 \\
\hline India & BSE Sensex 30 & Corea del Sur & KOSPI \\
\hline Indonesia & Jakarta SEC & España & IBEX 35 \\
\hline Irlanda & ISEQ Overall & Suecia & OMX Stockholm 30 \\
\hline Israel & TA 35 & Tailandia & SET \\
\hline Japón & Nikkei 225 & Tunez & Tunindex \\
\hline Jordania & MSCI Jordan & Turquia & BIST 100 \\
\hline Italia & FTSE MIB & Emiratos Arabes Unidos & ADX General \\
\hline Kazajstan & KASE & Reino Unido & FTSE 100 \\
\hline Libano & BLOM & Estados Unidos & S\&P 500 \\
\hline Malaysisa & KLCI & Vietnam & $\mathrm{VN}$ \\
\hline Mexico & S\&P IPC & & \\
\hline
\end{tabular}


Se utiliza la base de datos OxCGRT (Hale, Webster Petherick, Phillips y Kira, 2020b) para cuantificar la respuesta de los gobiernos a la crisis originada por COVID-19. La OXCGRT mide las respuestas de los gobiernos con tres índices principales: índice de rigor, índice de contención sanitaria y el índice de respuesta gubernamental. El índice de rigor registra información sobre medidas de distanciamiento social y se calcula a partir de 8 indicadores, incluido el cierre de escuelas, cierre del lugar de trabajo, cancelación de eventos públicos, restricciones a las reuniones, cierre del transporte público, requisitos de permanencia en el hogar, restricciones a la circulación interna y restricciones a viajar. El índice de contención sanitaria se construye a partir de 3 indicadores que representan las campañas de concientización pública, la política de pruebas y el rastreo de contactos. El índice de respuesta gubernamental se compone de los índices de restricción y del índice de contención sanitaria añadiendo dos indicadores adicionales, estos indicadores son el apoyo a los ingresos de la población por parte del gobierno y los programas de alivio de deuda para los hogares. Cada uno de los tres índices es una simple puntuación aditiva de los indicadores subyacentes, y se reajusta para variar de 0 a 100. Los índices tienen fines comparativos y no deben ser interpretado como una calificación de la idoneidad o eficacia de la respuesta del país ${ }^{2}$ a la pandemia COVID-19 (Hale, Petherick, Phillips y Webster 2020a). Así mismo la base de datos OxCGRT es el origen de los datos para las variables casos confirmados y decesos confirmados ambas calculadas como tasas de crecimiento.

Los datos para todas las variables son diarios exceptuando los días sábado y domingo, así como los días no laborales para cada país. Se elige el período del 2 de febrero al 28 de agosto de 2020. Seleccionamos este período porque se incluye los primeros casos confirmados por COVID-19 así como las respuestas de los gobiernos de cada país, las cuales se produjeron principalmente durante este período. Este intervalo incluye el seguimiento al comportamiento de la pandemia una vez que paso el pico de la primera ola (Solis, Franco-Paredes, Henao-Martínez, Krsak y Zimmer 2020). Este período de estudio a su vez se divide en catorce muestras dos por cada mes como se puede observar en la tabla 2. Por tanto, se consideran catorce sub períodos los cuales son utilizados para analizar el impacto de la pandemia en intervalos de tiempo más pequeños y encontrar los intervalos donde la pandemia COVID-19 tuvo mayor efecto sobre la volatilidad realizada.

Tabla 2. Período de tiempo para cada muestra

\begin{tabular}{|c|c|c|c|}
\hline Muestra & Período & Muestra & Período \\
\hline$I$ & $02 / 02 / 2020$ a $14 / 02 / 2020$ & $V I I I$ & $18 / 05 / 2020$ a $29 / 05 / 2020$ \\
\hline$I I$ & $17 / 02 / 2020$ a $28 / 02 / 2020$ & $I X$ & $01 / 06 / 2020$ a $12 / 06 / 2020$ \\
\hline$I I I$ & $02 / 03 / 2020$ a $13 / 03 / 2020$ & $X$ & $15 / 06 / 2020$ a $30 / 06 / 2020$ \\
\hline$I V$ & $16 / 03 / 2020$ a $31 / 03 / 2020$ & $X I$ & $01 / 07 / 2020$ a $15 / 07 / 2020$ \\
\hline$V$ & $01 / 04 / 2020$ a $15 / 04 / 2020$ & $X I I$ & $16 / 07 / 2020$ a $31 / 07 / 2020$ \\
\hline$V I$ & $16 / 04 / 2020$ a 30/04/2020 & $X I I I$ & $03 / 08 / 2020$ a $14 / 08 / 2020$ \\
\hline$V I I$ & $01 / 05 / 2020$ a $15 / 05 / 2020$ & $X I V$ & $17 / 08 / 2020$ a $28 / 08 / 2020$ \\
\hline
\end{tabular}

Fuente: Elaboración propia

\footnotetext{
${ }^{2}$ Más información sobre la composición de los índices: de rigor, contención sanitaria y de respuesta gubernamental se encuentra en el trabajo de Hale, Petherick, Phillips y Webster (2020a) y en la página: www.bsg.ox.ac.uk/covidtracker.
} 
Ramelli y Wagner (2020) sostienen que el período más importante en lo que respecta a la reacción del mercado a COVID-19 fue el comprendido entre el 20 de enero y el 20 de marzo de 2020. Asimismo, Hale, Petherick, Phillips y Webster (2020a) muestran que las curvas medias de respuesta de los gobiernos a nivel mundial se aplanaron, e incluso comenzaron a disminuir, a partir de mediados de abril. En la tabla 3 se pueden observar las abreviaturas de las variables y su unidad de medida.

Tabla 3. Abreviaturas y unidad de medida.

\begin{tabular}{|l|c|l|}
\hline Variable & & \\
\hline Volatilidad realizada & $v r$ & Número \\
\hline Casos confirmados & $g c c$ & Tasa de crecimiento \\
\hline Decesos confirmados & $g d c$ & Tasa de crecimiento \\
\hline Índice de restricción o rigor & $i s$ & Número \\
\hline Índice de contención sanitaria & $i c h$ & Número \\
\hline Índice de respuesta gubernamental & $i r g$ & Número \\
\hline
\end{tabular}

Fuente: Elaboración propia

En la tabla 4 podemos observar las estadísticas básicas para el período del 2 de febrero al 28 de agosto. Las tablas con las estadísticas básicas de las catorce muestras se encuentran en el apéndice con el nombre tabla $1 \mathrm{a}$.

Tabla 4. Estadísticas básicas del 2 de febrero al 28 de agosto

\begin{tabular}{|l|c|c|c|c|c|c|}
\hline & $\boldsymbol{v r}$ & $\boldsymbol{g c c}$ & $\boldsymbol{g d c}$ & $\boldsymbol{i s}$ & $\boldsymbol{i c h}$ & $\boldsymbol{i r g}$ \\
\hline Media & 0.01 & 0.09 & 0.07 & 56.42 & 57.93 & 56.62 \\
\hline Máximo & 1.636 & 43 & 6.5 & 100 & 100 & 96.15 \\
\hline Mínimo & .00003 & 0.00 & 0.00 & 0.00 & 0.00 & 0.00 \\
\hline Desviación estándar & 0.02 & 0.62 & 0.27 & 27.10 & 24.71 & 24.04 \\
\hline Observaciones & 8977 & 8663 & 7478 & 9740 & 9738 & 9738 \\
\hline
\end{tabular}

Fuente: Elaboración propia

Con los datos resultantes se construyen catorce paneles de datos no balanceados.

\section{Metodología}

Con el fin de analizar las cinco premisas en estudio las cuales son: 1) el índice de rigor tendrá un efecto positivo sobre la volatilidad en los índices bursátiles, 2) el nexo entre el índice de contención sanitaria y la volatilidad en los índices bursátiles será negativo 3) la relación entre el índice de respuesta gubernamental y la volatilidad es negativa 4) el número de casos confirmados incrementa la volatilidad y de igual manera 5) el número de decesos confirmados tiene un efecto positivos sobre la volatilidad. El primer paso es establecer el modelo de regresión para panel de datos para cada una de las muestras. En las catorce muestras se emplean las siguientes variables: la volatilidad realizada (vr) como variable dependiente y como variables independientes se utilizan: casos confirmados 
$(g c c)$, decesos confirmados $(g d c)$, índice de restricción (is), índice del estado de salud de la población (ich) e índice de respuesta gubernamental (irg). El método de estimación que se utiliza es el método de Mínimos Cuadrados Ordinarios con efectos fijos de dos vías. La ecuación (2) muestra el modelo para todas las muestras:

$$
v r_{i, t}=c+g c c_{i, t}+g d c_{i, t}+i s_{i, t}+i c h_{i, t}+i r g_{i, t}+u_{i, t}
$$

Donde $i$ representa el país, $t$ representa el tiempo. Las muestras abarcan desde el 2 de febrero al 28 de agosto de 2020. En este método se asume que el término de error $u_{i, t}$ es un término de perturbación puro. Sin embargo, cuando se considera un método de dos vías el término de error está compuesto por: efectos individuales no observables, efectos no observables en el tiempo y un término de perturbación pura. Por tanto, el término de error se puede expresar de la siguiente manera:

$$
u_{i t}=\theta_{i}+\tau_{t}+\varepsilon_{i t}
$$

Donde $\theta_{i}$ y $\tau_{t}$ son los efectos individuales no observables y efectos no observables en el tiempo respectivamente. Si $\theta_{i}$ o $\tau_{t}$ es diferente de cero entonces se trata de un modelo de una vía para el componente de error. Si ambos $\theta_{i}$ y $\tau_{t}$ son diferentes de cero se considera un modelo de dos vías para el componente de error. Existen dos métodos para estimar el término de error de la ecuación (3) estos son: efectos fijos y efectos aleatorios. La base de datos que se considera para este análisis está conformada por un índice bursátil para cada país. Y cada país tiene características específicas propias que no se pueden observar directamente los cuales podrían influir sobre la volatilidad realizada de cada índice. Estas características pueden ser de tipo: cultural y religioso entre otros. Así mismo existen ciertos factores que ocurren en el tiempo como huelgas y desastres naturales que tampoco se pueden observar directamente. Por tanto, se considera un modelo de efectos fijos de dos vías el cual considera la inclusión de dos variables dummies una para las características específicas de cada país y otra para los efectos del tiempo (Kaizoji y Miyano, 2016). El modelo se expresa de la siguiente forma:

$$
v r_{i t}=c+g c c_{i, t}+g d c_{i, t}+i s_{i, t}+i r g_{i, t}+\theta_{i}+\tau_{t}+\varepsilon_{i t}
$$

Por otro lado, los resultados de la prueba Hausman de Efectos Aleatorios Correlacionados (período) presenta evidencia en favor del uso de efectos fijos de dos vías para las muestras $I, I I, I I I$, $I V, V, V I$ y $V I I$ como se puede observar en las tablas 5 y 6 . Así mismo, esta prueba indica que para las muestras VIII, IX,X,XI,XII,XIII, y XIV el método de estimación adecuado es el de Mínimos Cuadrados Generalizados Estimados (EGLS) con efectos aleatorios de una vía (período). El método considera que en algunos casos no se conoce la matriz de covarianza $(\Psi)$. Por lo tanto, esta matriz debe ser estimada. Para obtener dicha matriz se considera el siguiente modelo:

$$
y_{t}=\beta X+u_{t}
$$

Donde $y_{t}$ es la variable dependiente, $\beta$ es el coeficiente a estimar, $X$ es una matriz $n * p$ de variables independientes y $u_{t}$ es el término de error. Con $t=1, \ldots . N$. Al tomar la media de todas las 
observaciones de la variable dependiente y el término de error. El modelo se transforma en la siguiente expresión:

$$
\bar{y}=\beta X+\bar{u}
$$

Donde $\bar{y}$ y $u$ son los promedios de la variable dependiente y el término de error respectivamente. Por lo tanto, el estimador imparcial de $\Psi$ estará dado por la siguiente ecuación:

$$
\widehat{\Psi}=(N-1)^{-1} \sum_{t=1}^{N}\left(y_{t}-\bar{y}\right)\left(y_{t}-\bar{y}\right)^{T}
$$

Se conoce que $\widehat{\Psi}$ es un estimado de $\Psi$ entonces el estimado de Mínimos Cuadrados Generalizados para $\beta$ esta dado por la siguiente ecuación (Sen y Srivastava, 2012):

$$
\beta_{E G L S}=\left(X^{T} \hat{\Sigma}^{-1} X\right)^{-1} X^{T} \widehat{\Sigma}^{-1} \bar{y}
$$

En este método al igual que en el método de Mínimos Cuadrados Ordinarios se asume que el término de error está compuesto por: efectos individuales no observables, efectos no observables en el tiempo y el término de perturbación pura. Aunque, en este caso solo se considera el modelo de una vía para el componente de error, es decir solo se emplean efectos no observables en el tiempo (período). Por tanto, el modelo a estimar se expresa de la siguiente manera:

$$
v r_{i t}=c+g c c_{i, t}+g d c_{i, t}+i s_{i, t}+i r g_{i, t}+\tau_{t}+\varepsilon_{i t}
$$

Se utiliza el modelo de la ecuación (9) para estimar las muestras $V I, V I I, V I I I, X, X I, X I I$, y $X I V$ con el método de Mínimo Cuadrados Generalizados Estimados (EGLS) con efectos aleatorios de una vía (período) los resultados se muestran en la tabla 1c que se encuentra en el anexo.

La ventaja de usar esta metodología es que permite conocer de manera específica en el tiempo cuando los factores estudiados tienen mayor efecto sobre la volatilidad realizada. En cuanto a las técnicas econométricas se usa como estimación base el método de Minimos Cuadrdados Ordinarios con efectos de dos vías para controlar el impacto de la heterogeneidad no observada. Al incorporar la técnica Mínimos Cuadrados Generalizados Estimados (EGLS) con efectos aleatorios de una vía (período) se permite controlar los problemas de autocorrelación y heterocedasticidad. Además, se justifica el uso del modelo de efectos aleatorios por las siguientes razones 1) por el resultado de la prueba de prueba Hausman de Efectos Aleatorios Correlacionados (período) 2) por que el interés se centra en la población de la que se extrae la muestra, más que en las características no observadas específicas del país en sí.

\section{Resultados}

Se realizaron catorce estimaciones dos por cada mes. Se empleó el método Mínimos Cuadraos Ordinarios con efectos fijos de dos vías para realizar las estimaciones. Los resultados de las muestras $I, I I, I I I, I V, V, V I$ y VII se muestran en la tabla 5 . 
Tabla 5. Resultados de las muestras $I$, II, $I I I, I V, V, V I$ y $V I I$ con el método de Mínimos Cuadraos Ordinarios con efectos fijos de dos vías.

\begin{tabular}{|c|c|c|c|c|c|c|c|}
\hline & $I$ & $I I$ & $I I I$ & $I V$ & $\boldsymbol{V}$ & $\boldsymbol{V I}$ & $\boldsymbol{V I I}$ \\
\hline$c$ & -0.0190 & -0.0063 & 0.0195 & $0.0583^{* * *}$ & $0.0463^{* * *}$ & $0.037^{* * *}$ & 0.0056 \\
\hline$g c c$ & -0.0002 & -0.0038 & -0.0034 & $0.0093^{* *}$ & -0.0037 & -0.0057 & 0.0060 \\
\hline$g d c$ & 0.0101 & 0.0027 & 0.0028 & -0.0007 & 0.0007 & -0.0034 & -0.0141 \\
\hline is & -0.0005 & - & & & & & \\
\hline ich & 0.3116 & $0.0015^{* * *}$ & -0.0083 & -0.0003 & $-0.0010^{* *}$ & 0.0001 & -.00004 \\
\hline irg & -0.3669 & -0.0002 & 0.0090 & $-0.0007^{*}$ & 0.0002 & -0.0003 & 0.0005 \\
\hline Estadísticas & & & & & & & \\
\hline$R-$ cuadrado & 0.93 & 0.73 & 0.83 & 0.64 & 0.56 & 0.50 & 0.62 \\
\hline $\begin{array}{c}\text { Estadístico } \\
\text { Durbin } \\
\text { Watson }\end{array}$ & 1.83 & 2.10 & 1.80 & 1.99 & 1.82 & 1.86 & 2.09 \\
\hline $\begin{array}{c}\text { Prueba Hausman } \\
\text { de Efectos } \\
\text { Aleatorios } \\
\begin{array}{c}\text { Correlacionados } \\
\text { (período) }\end{array}\end{array}$ & 0.00 & 0.00 & 0.00 & 0.00 & 0.04 & 0.19 & 0.12 \\
\hline \begin{tabular}{c} 
Observaciones \\
\hline
\end{tabular} & 27 & 56 & 115 & 511 & 554 & 620 & 572 \\
\hline
\end{tabular}

Fuente: Elaboración propia

Los resultados para la muestra II exhiben que el índice de rigor y el índice de contención sanitaria son estadísticamente significativas el primero al 5\% y el segundo al 1\%. El índice de rigor muestra un signo negativo lo que significa que un aumento de una unidad en esta variable apunta a una reducción de la volatilidad de .00009 , la variable $i c h$ muestra signo positivo lo que indica que un aumento de una unidad en esta variable signficaría un aumento de la volatilidad de .0015. Los resultados de la muestra $I V$ indican que la variable $g c c$ es estadísticamente significativa al 5\% cuando gcc aumenta uno por ciento la volatilidad aumenta .0093, mientras que la variable $\mathrm{irg}$ es estadísticamente significativa al $10 \%$ un aumento de una unidad de esta variable significa una disminución de .00007 en la volatilidad. Por otro lado, la variable is es estadísticamente significativa al $10 \%$ en la muestra $V$, así mismo la variable $i c h$ es estadísticamente significativa al $5 \%$. Un aumento de una unidad para la variable is provoca un aumento de .0005 en la volatilidad, por otro lado, un aumento de una unidad en la variable $i c h$ se traduce en una disminución de .001 en la volatilidad. Para las muestras I, III, VI y VII los resultados muestran que ninguna variable es estadísticamente significativa. Los resultados de las muestras VIII, IX, X, XI, XII, XIII y XIV se pueden observar en la tabla 6. 
Tabla 6. Resultados de las muestras VIII, IX,X,XI, XII, XIII y XIV con el método de Mínimos Cuadraos Ordinarios con efectos fijos de dos vías.

\begin{tabular}{|c|c|c|c|c|c|c|c|}
\hline & VIII & $I X$ & $X$ & $X I$ & $X I I$ & XIII & $X I V$ \\
\hline$c$ & 0.0194 & 0.0132 & 0.0130 & $0.0284^{* *}$ & $0.0264^{*}$ & -0.0097 & 0.0028 \\
\hline$g c c$ & 0.0326 & $0.0303^{*}$ & 0.0262 & 0.0084 & 0.0105 & 0.0181 & -0.0059 \\
\hline$g d c$ & 0.0121 & 0.0100 & 0.0159 & 0.0084 & -0.0024 & -0.011 & -0.0078 \\
\hline is & -0.0007 & -0.00006 & 0.0003 & 0.0002 & 0.0003 & -0.0005 & -0.0007 \\
\hline ich & $\begin{array}{r}- \\
0.0003\end{array}$ & 0.0005 & $\begin{array}{r}- \\
0.0003\end{array}$ & 0.0009 & 0.0013 & 0.0001 & 0.0010 \\
\hline irg & $0.0011^{* *}$ & -0.0004 & 0.00008 & -0.0014 & -0.0012 & 0.0008 & -0.0002 \\
\hline Estadísticas & & & & & & & \\
\hline$R$ - cuadrado & 0.55 & 0.66 & 0.62 & 0.50 & 0.46 & 0.56 & 0.56 \\
\hline $\begin{array}{c}\text { Estadístico Durbin } \\
\text { Watson }\end{array}$ & 1.82 & 1.99 & 1.87 & 2.19 & 2.00 & 1.61 & 1.95 \\
\hline $\begin{array}{c}\text { Prueba Hausman } \\
\text { de Efectos } \\
\text { Aleatorios } \\
\text { Correlacionados } \\
\text { (período) }\end{array}$ & 0.57 & 0.03 & 0.75 & 0.27 & 0.27 & 0.00 & 0.14 \\
\hline Observaciones & 539 & 582 & 710 & 649 & 698 & 592 & 595 \\
\hline
\end{tabular}

Fuente: Elaboración propia

Los resultados para la variable irg muestran que es estadísticamente significativa al 5\% en las muestras VIII y XII, también es estadísticamente significativa al $10 \%$ en la muestra XI. Un aumento de una unidad en esta variable significa un aumento en la volatilidad en la muestra VIII de .0011 y una disminución en la volatilidad en la muestra XI y XII de .0014 y .0012 respectivamente. En la muestra VIII la variable is es estadísticamente significativa al 10\%. Un aumento de una unidad en esta variable significa una disminución de .0007 en la volatilidad realizada. La variable gcc es estadísticamente significativa al $10 \%$ en la muestra $I X$, un aumento de una unidad en esta variable significa un aumento en la volatilidad de .0303. En las muestras X, XIII y XIV las variables utilizadas no son estadísticamente significativas.

Por otro lado, dados los resultados de la prueba Hausman de Efectos Aleatorios Correlacionados indican que se debe realizar la estimación empleando el método de Mínimos Cuadrados Generalizados Estimados (EGLS) con efectos aleatorios de una vía (período) en las muestras $V I, V I I, V I I I, X, X I, X I I$, y $X I V$. Los resultados de las estimaciones para las muestras antes mencionadas se muestran en la tabla 1c la cual se encuentra en el anexo, los resultados de estas muestran apuntan a que no existe evidencia de una relación entre las variables empleadas en el estudio y la volatilidad realizada. Debido a que los resultados de todas las muestras presentan índices de correlación que en ningún caso supera .07.

En general los resultados obtenidos presentan evidencia a favor de la existencia de una relación estadística entre la volatilidad y las medidas para contener el COVID-19 en las muestras II, 
$I V$ y $V$. Sin embargo, dada la evidencia obtenida no podemos determinar el sentido de la relación. Específicamente la variable is muestra evidencia a favor de la hipótesis de una relación positiva entre el índice de rigor y la volatilidad realizada en los principales índices bursátiles en la muestra $V$; mientras que en la muestra II se encuentra evidencia en contra de esta hipótesis. De manera similar la variable ich muestra evidencia a favor de la hipótesis de una relación negativa entre el índice de contención sanitaria y la volatilidad en la muestra $V$ y en contra de la hipótesis en la muestra II. En cuanto a la variable irg se encuentra evidencia a favor de la hipótesis de una relación negativa entre el índice de respuesta gubernamental y la volatilidad en la muestra $I V$.

En cuanto a los efectos de la pandemia causada por COVID.19 los resultados muestran que la variable $g c c$ muestra evidencia a favor de una relación positiva entre el número de casos confirmados y la volatilidad en las muestras $I V$ y $I X$. Mientras que la variable $g d c$ no es estadísticamente significativa para ninguna de las muestras.

Los resultados obtenidos en esta investigación coinciden con el trabajo de Zaremba, Kizys, Aharon y Demir (2020) ya que ellos encuentran que las intervenciones gubernamentales afectan de manera significativa y robusta la volatilidad de los mercados bursátiles internacionales sobre todo en los meses de febrero y marzo. Sin embargo, los resultados obtenidos en este estudio difieren con respecto al trabajo de Zaremba, Kizys, Aharon y Demir (2020) dado que en el presente estudio no podemos determinar el sentido de la relación.

Por otro lado, los resultados obtenidos en el trabajo de Albulescu (2020b) le permite concluir que los efectos del brote de COVID-19 (casos confirmados y decesos confirmados) aumentan la volatilidad realizada en el índice S\&P 500. Este estudio encuentra que los casos confirmados de COVID-19 solo tienen un efecto positivo sobre la volatilidad en la muestra $I V$ mientras que la variable decesos confirmados no es estadísticamente significativa en ninguna muestra.

\section{Conclusiones}

Se estudió la relación entre los efectos de la pandemia COVID-19 y la volatilidad realizada. Además, se analizó el posible nexo entre las medidas de mitigación y contención adoptadas por los gobiernos y la volatilidad realizada. El período en consideración abarca del 2 de febrero al 28 de agosto del 2020. Este período a su vez se dividió en catorce muestras dos por cada mes, lo que dio origen a los catorce paneles no balanceados usados en las estimaciones. Las estimaciones se realizaron con las técnicas econométricas de Mínimos Cuadrados Ordinarios con efectos fijos de dos vías y el método de Mínimo Cuadrados Generalizados Estimados (EGLS) con efectos aleatorios de una vía (período).

Se encontró evidencia que apoya la existencia de una relación estadística significativa entre las medidas de contención y mitigación y la volatilidad realizada en los meses de febrero y marzo, sin embargo, no se encontró uniformidad en los resultados. Por otro lado, no se encontró evidencia suficiente a favor de la existencia de una relación positiva entre los efectos de la pandemia COVID-19 y la volatilidad realizada ya que, de catorce muestras en solo dos se encontró evidencia de un nexo positivo y estadísticamente significativo. Mientras que la variable gdc no es estadísticamente significativa en ninguna muestra. Por tanto, básicamente se encontró coincidencia con el estudio de Zaremba, Kizys, Aharon y Demir 2020 en el sentido de que las medidas que adoptaron los gobiernos para combatir los efectos de la pandemia afectan de manera significativa a la volatilidad en los mercados financieros sobre todo en los meses de febrero y marzo. 
Dado los resultados obtenidos se sugiere a los gobiernos alrededor del mundo tener en consideración que además del impacto económico que tienen las medidas de mitigación y contención, estas también tienen un efecto importante sobre los mercados financieros. Ya que estas intervenciones pueden aumentar la incertidumbre o reducirla y por tanto afectar de manera significativa la volatilidad de los mercados financieros. En caso de un aumento de la volatilidad puede provocar episodios de ventas generalizadas de activos de riesgo. Además de que altos niveles de volatilidad también puede traducirse en un mayor costo de capital. Para futuras investigaciones se sugiere analizar el papel individual que juegan las diversas medidas de contención utilizando un marco temporal más extenso.

\section{Referencias}

[1] Al-Awadhi, A. M., Alsaifi, K., Al-Awadhi, A. y Alhammadi, S. (2020). Death and contagious infectious diseases: Impact of the COVID-19 virus on stock market returns. Journal of Behavioral and Experimental Finance, 27, 100326. http://dx.doi.org/10.1016/j.jbef.2020.100326.

[2] Albulescu, C. (2020a). Coronavirus and Financial Volatility: 40 Days of Fasting and Fear. SSRN Electronic Journal. http://dx.doi.org/10.2139/ssrn.3550630.

[3] Albulescu, C. T. (2020b). COVID-19 and the United States financial markets' volatility. Finance Research Letters, 101699. http://dx.doi.org/10.1016/j.frl.2020.101699.

[4] Albuquerque, R., Koskinen, Y., Yang, S. y Zhang, C. (2020). Resiliency of Environmental and Social Stocks: An Analysis of the Exogenous COVID-19 Market Crash. The Review of Corporate Finance Studies, 9(3), 593-621. http://dx.doi.org/10.1093/rcfs/cfaa011.

[5] Alfaro, L., Chari, A., Greenland, A. y Schott, P. (2020). Aggregate and Firm-Level Stock Returns During Pandemics, in Real Time. http://dx.doi.org/10.3386/w26950.

[6] Ali, M., Alam, N. y Rizvi, S. A. R. (2020). Coronavirus (COVID-19) - An epidemic or pandemic for financial markets. Journal of Behavioral and Experimental Finance, 27, 100341. http://dx.doi.org/10.1016/j.jbef.2020.100341.

[7] Ashraf, B. N. (2020a). Stock markets' reaction to COVID-19: Cases or fatalities? Research in International Business and Finance, 54, 101249. http://dx.doi.org/10.1016/j.ribaf.2020.101249

[8] Ashraf, B. N. (2020b). Economic impact of government interventions during the COVID-19 pandemic: International evidence from financial markets. Journal of Behavioral and Experimental Finance, 27, 100371. http://dx.doi.org/10.1016/j.jbef.2020.100371

[9] Baek, S., Mohanty, S. K. y Glambosky, M. (2020). COVID-19 and stock market volatility: An industry level analysis. Finance Research Letters, 37, 101748.

[10] Baker, S., Bloom, N. y Terry, S. (2020). Using Disasters to Estimate the Impact of Uncertainty. NBER Working paper. https://doi.org/10.3386/w27167

[11] Baker, S., Bloom, N., Davis, S., Kost, K., Sammon, M. y Viratyosin, T. (2020). The Unprecedented Stock Market Impact of COVID-19. http://dx.doi.org/10.3386/w26945.

[12] Chun, D., Cho, H. y Ryu, D. (2020). Economic indicators and stock market volatility in an emerging economy. Economic Systems, 44 (2), 100788. http://dx.doi.org/10.1016/j.ecosys.2020.100788.

[13] Conlon, T., Corbet, S. y McGee, R. (2020). Are Cryptocurrencies a Safe Haven for Equity Markets? An International Perspective from the COVID-19 Pandemic. SSRN Electronic Journal. http://dx.doi.org/10.2139/ssrn.3601045. 
[14] Conlon, T. y McGee, R. (2020). Safe Haven or Risky Hazard? Bitcoin during the Covid-19 Bear Market. SSRN Electronic Journal. http://dx.doi.org/10.2139/ssrn.3560361.

[15] Corbet, S., Hou, Y., Hu, Y., Lucey, B. y Oxley, L. (2020a). Aye Corona! The contagion effects of being named Corona during the COVID-19 pandemic. Finance Research Letters, 101591. http://dx.doi.org/10.1016/j.frl.2020.101591.

[16] Corbet, S., Larkin, C. J. y Lucey, B. M. (2020b). The Contagion Effects of the COVID-19 Pandemic: Evidence from Gold and Cryptocurrencies. SSRN Electronic Journal.

[17] Dietrich, A., Kuester, K., Müller, G. J. y Schoenle, R. (2020). News and Uncertainty about COVID-19: Survey Evidence and Short-Run Economic Impact. SSRN Electronic Journal. https://doi.org/10.2139/ssrn.3573123

[18] Donthu, N. y Gustafsson, A. (2020). Effects of COVID-19 on business and research. Journal of Business Research, 117, 284-289. https://doi.org/10.1016/j.jbusres.2020.06.008

[19] Dunford, D., Dale, B., Stylianou N., Lowther, E., Ahmed, M. y Arenas, I. (2020) Coronavirus: The world in lockdown in maps and charts. Disponible en https://www.bbc.com/news/world-52103747

[20] Edwards, T. y Lazzara, C. J. (2016) Realized Volatility Indices: Measuring Market Risk. Disponible en https://www.spglobal.com/spdji/en/documents/research/research-realized-volatility-indicesmeasuring-market-risk.pdf?force_download=true

[21] Goodell, J. W. y Goutte, S. (2020). Co-Movement of COVID-19 and Bitcoin: Evidence from Wavelet Coherence Analysis. SSRN Electronic Journal. http://dx.doi.org/10.2139/ssrn.3597144.

[22] Hale, T., Petherick, A., Phillips, T. y Webster, S. (2020a). Variation in government responses to COVID19. Blavatnik school of government working paper, 31 .

[23] Hale, T., Webster, S., Petherick, A., Phillips, T. y Kira, B. (2020b). Oxford covid-19 government response tracker. Blavatnik School of Government, 25.

[24] Haroon, O., y Rizvi, S. A. R. (2020). COVID-19: Media coverage and financial markets behavior-A sectoral inquiry. Journal of Behavioral and Experimental Finance, 27, 100343. Available at: http://dx.doi.org/10.1016/j.jbef.2020.100343.

[25] Kaizoji, T., y Miyano, M. (2019). Stock market crash of 2008: an empirical study of the deviation of share prices from company fundamentals. Applied Economics Letters, 26(5), 362-369. https://doi.org/10.1080/13504851.2018.1486004

[26] Ludvigson, S., Ma, S. y Ng, S. (2020). Covid19 and the Macroeconomic Effects of Costly Disasters. https://doi.org/10.3386/w26987

[27] Norouzi, N., Zarazua de Rubens, G., Choupanpiesheh, S. y Enevoldsen, P. (2020). When pandemics impact economies and climate change: Exploring the impacts of COVID-19 on oil and electricity demand in China. Energy Research \& Social Science, 68, 101654. https://doi.org/10.1016/j.erss.2020.101654

[28] Onali, E. (2020). COVID-19 and Stock Market Volatility. SSRN Electronic Journal. doi:10.2139/ssrn.3571453. http://dx.doi.org/10.2139/ssrn.3571453.

[29] Ramelli, S. y Wagner, A. F. (2020). Feverish stock price reactions to COVID-19. The Review of Corporate Finance Studies, 9(3), 622-655. https://doi.org/10.1093/rcfs/cfaa012

[30] Schell, D., Wang, M. y Huynh, T. L. D. (2020). This time is indeed different: A study on global market reactions to public health crisis. Journal of Behavioral and Experimental Finance, 27, 100349. http://dx.doi.org/10.1016/j.jbef.2020.100349.

[31] Sen, A. y Srivastava, M. (2012). Regression analysis: theory, methods, and applications. Springer Science \& Business Media.

[32] Sharif, A., Aloui, C. y Yarovaya, L. (2020). COVID-19 Pandemic, Oil Prices, Stock Market and Policy Uncertainty Nexus in the US Economy: Fresh Evidence from the Wavelet-Based Approach. SSRN Electronic Journal. http://dx.doi.org/10.2139/ssrn.3574699. 
[33] Solis, J., Franco-Paredes, C., Henao-Martínez, A. F., Krsak, M., y Zimmer, S. M. (2020). Structural vulnerability in the US revealed in three waves of COVID-19. The American journal of tropical medicine and hygiene, 103(1), 25-27.

[34] Zhang, D., Hu, M. y Ji, Q. (2020). Financial markets under the global pandemic of COVID-19. Finance Research Letters, 36, 101528. http://dx.doi.org/10.1016/j.frl.2020.101528.

[35] Zaremba, A., Kizys, R., Aharon, D. Y. y Demir, E. (2020). Infected Markets: Novel Coronavirus, Government Interventions, and Stock Return Volatility around the Globe. Finance Research Letters, 35, 101597.

\section{Anexo}

Tabla 1a. Estadísticas básicas de las muestras $I, I I, I I I, I V, V, V I$ y $V I I$

\begin{tabular}{|c|c|c|c|c|c|c|}
\hline \multicolumn{7}{|l|}{ Muestra $I$} \\
\hline & $v r$ & $g c c$ & $g d c$ & is & ich & $\operatorname{irg}$ \\
\hline Media & 0.01 & 0.10 & 0.07 & 11.27 & 13.82 & 11.75 \\
\hline Máximo & 0.11 & 1.14 & 0.69 & 77.31 & 75.38 & 63.78 \\
\hline Mínimo & 0.0001 & 0.00 & 0.00 & 0.00 & 0.00 & 0.00 \\
\hline $\begin{array}{l}\text { Desviación } \\
\text { estándar }\end{array}$ & 0.007 & 0.21 & 0.15 & 13.75 & 13.94 & 11.80 \\
\hline Observaciones & 622 & 224 & 28 & 650 & 650 & 650 \\
\hline \multicolumn{7}{|l|}{ Muestra II } \\
\hline Media & 0.01 & 0.35 & 0.25 & 13.80 & 16.89 & 14.42 \\
\hline Máximo & 0.11 & 43.00 & 6.00 & 81.02 & 81.44 & 68.91 \\
\hline Mínimo & 0.0006 & 0.00 & 0.00 & 0.00 & 0.00 & 0.00 \\
\hline $\begin{array}{l}\text { Desviación } \\
\text { estándar }\end{array}$ & 0.01 & 2.74 & 0.87 & 16.02 & 16.27 & 14.03 \\
\hline Observaciones & 614 & 263 & 58 & 650 & 650 & 650 \\
\hline \multicolumn{7}{|l|}{ Muestra III } \\
\hline Media & 0.03 & 0.65 & 0.34 & 23.44 & 27.86 & 24.29 \\
\hline Máximo & 0.26 & 18.00 & 4.60 & 85.19 & 84.85 & 71.79 \\
\hline Mínimo & 0.0002 & 0.00 & 0.00 & 0.00 & 0.00 & 0.00 \\
\hline $\begin{array}{l}\text { Desviación } \\
\text { estándar }\end{array}$ & 0.03 & 1.54 & 0.62 & 17.76 & 16.77 & 14.88 \\
\hline Observaciones & 624 & 392 & 117 & 650 & 650 & 650 \\
\hline \multicolumn{7}{|l|}{ Muestra IV } \\
\hline Media & 0.04 & 0.33 & 0.45 & 67.51 & 65.27 & 60.04 \\
\hline Máximo & 0.17 & 8.00 & 6.50 & 100.00 & 96.97 & 91.03 \\
\hline Mínimo & 0.00003 & 0.0002 & 0.0009 & 2.78 & 6.82 & 5.77 \\
\hline $\begin{array}{l}\text { Desviación } \\
\text { estándar }\end{array}$ & 0.02 & 0.51 & 0.77 & 19.79 & 16.99 & 16.12 \\
\hline Observaciones & 734 & 764 & 532 & 780 & 780 & 780 \\
\hline \multicolumn{7}{|l|}{ Muestra $V$} \\
\hline Media & 0.02 & 0.10 & 0.16 & 80.94 & 77.36 & 74.13 \\
\hline Máximo & 0.08 & 0.88 & 1.50 & 100.00 & 100.00 & 96.15 \\
\hline Mínimo & 0.0005 & 0.0005 & 0.0002 & 38.89 & 40.91 & 42.31 \\
\hline $\begin{array}{l}\text { Desviación } \\
\text { estándar }\end{array}$ & 0.01 & 0.11 & 0.20 & 12.83 & 11.65 & 10.60 \\
\hline Observaciones & 590 & 715 & 676 & 715 & 715 & 715 \\
\hline
\end{tabular}


DOI: https://doi.org/10.21919/remef.v16i3.605

\begin{tabular}{|l|c|c|c|c|c|c|}
\hline Muestra VI & & & & & & \\
\hline Media & 0.02 & 0.05 & 0.06 & 80.00 & 77.24 & 75.20 \\
\hline Máximo & 0.10 & 0.87 & 2.00 & 100.00 & 100.00 & 96.15 \\
\hline Mínimo & 0.0001 & 0.00002 & 0.0002 & 43.52 & 44.70 & 45.51 \\
\hline $\begin{array}{l}\text { Desviación } \\
\text { estándar }\end{array}$ & 0.01 & 0.07 & 0.10 & 12.66 & 11.17 & 9.87 \\
\hline Observaciones & 712 & 780 & 744 & 780 & 780 & 780 \\
\hline Muestra VII & & & & & & \\
\hline Media & 0.01 & 0.03 & 0.03 & 75.73 & 74.56 & 72.95 \\
\hline Máximo & 0.08 & 0.45 & 0.50 & 96.30 & 96.97 & 93.59 \\
\hline Mínimo & 0.0006 & 0.00001 & 0.001 & 36.11 & 40.91 & 46.15 \\
\hline $\begin{array}{l}\text { Desviación } \\
\text { estándar }\end{array}$ & 0.009 & 0.04 & 0.04 & 12.75 & 10.83 & 9.28 \\
\hline Observaciones & 602 & 715 & 682 & 715 & 715 & 715 \\
\hline
\end{tabular}

Fuente: Elaboración propia

Tabla 1b. Estadísticas básicas de las muestras VIII, IX, X, XI, XII, XIII y XIV

\begin{tabular}{|c|c|c|c|c|c|c|}
\hline \multicolumn{7}{|l|}{ Muestra VIII } \\
\hline & $v r$ & $g c c$ & $g d c$ & is & ich & irg \\
\hline Media & 0.01 & 0.02 & 0.02 & 71.79 & 71.80 & 70.58 \\
\hline Máximo & 1.63 & 1.29 & 0.35 & 96.30 & 93.94 & 88.46 \\
\hline Mínimo & 0.0005 & 0.00001 & 0.00003 & 31.48 & 33.33 & 39.74 \\
\hline $\begin{array}{l}\text { Desviación } \\
\text { estándar }\end{array}$ & 0.06 & 0.06 & 0.03 & 14.37 & 12.26 & 10.28 \\
\hline Observaciones & 623 & 715 & 682 & 715 & 715 & 715 \\
\hline \multicolumn{7}{|l|}{ Muestra $I X$} \\
\hline Media & 0.01 & 0.02 & 0.02 & 64.39 & 66.69 & 66.58 \\
\hline Máximo & 0.08 & 0.26 & 0.61 & 93.52 & 87.12 & 85.26 \\
\hline Mínimo & 0.00008 & 0.00001 & 0.00003 & 22.22 & 29.55 & 36.54 \\
\hline $\begin{array}{l}\text { Desviación } \\
\text { estándar }\end{array}$ & 0.01 & 0.03 & 0.04 & 16.35 & 13.86 & 11.71 \\
\hline Observaciones & 673 & 715 & 682 & 715 & 715 & 715 \\
\hline \multicolumn{7}{|l|}{ Muestra $X$} \\
\hline Media & 0.01 & 0.02 & 0.02 & 60.11 & 63.45 & 63.86 \\
\hline Máximo & 0.26 & 89.81 & 87.12 & 84.62 & 0.26 & 89.81 \\
\hline Mínimo & 0.00003 & 22.22 & 30.30 & 33.97 & 0.00003 & 22.22 \\
\hline $\begin{array}{l}\text { Desviación } \\
\text { estándar }\end{array}$ & 0.03 & 17.92 & 15.18 & 12.63 & 0.03 & 17.92 \\
\hline Observaciones & 744 & 780 & 744 & 780 & 780 & 780 \\
\hline \multicolumn{7}{|l|}{ Muestra XI } \\
\hline Media & 0.01 & 0.02 & 0.01 & 57.79 & 61.68 & 62.32 \\
\hline Máximo & 0.09 & 0.88 & 1.00 & 92.59 & 88.26 & 86.22 \\
\hline Mínimo & 0.001 & 0.00005 & 0.00007 & 22.22 & 30.30 & 37.18 \\
\hline $\begin{array}{l}\text { Desviación } \\
\text { estándar }\end{array}$ & 0.008 & 0.04 & 0.04 & 17.53 & 14.96 & 12.39 \\
\hline Observaciones & 678 & 715 & 684 & 715 & 714 & 714 \\
\hline \multicolumn{7}{|l|}{ Muestra $X I I$} \\
\hline Media & 0.01 & 0.01 & 0.01 & 57.64 & 61.72 & 62.43 \\
\hline Máximo & 0.06 & 0.20 & 1.00 & 94.44 & 92.42 & 87.82 \\
\hline Mínimo & 0.0007 & 0.0002 & 0.00007 & 22.22 & 30.30 & 37.18 \\
\hline $\begin{array}{l}\text { Desviación } \\
\text { estándar }\end{array}$ & 0.008 & 0.02 & 0.05 & 17.47 & 14.73 & 12.29 \\
\hline
\end{tabular}


REMEF (The Mexican Journal of Economics and Finance)

Volatilidad y COVID-19: evidencia empírica internacional

\begin{tabular}{|c|c|c|c|c|c|c|}
\hline Observaciones & 785 & 845 & 819 & 845 & 844 & 844 \\
\hline \multicolumn{7}{|l|}{ Muestra XIII } \\
\hline Media & 0.01 & 0.01 & 0.01 & 57.84 & 61.80 & 62.61 \\
\hline Máximo & 0.09 & 0.22 & 0.40 & 94.44 & 92.42 & 87.82 \\
\hline Mínimo & 0.001 & 0.0001 & 0.00002 & 22.22 & 30.30 & 29.49 \\
\hline $\begin{array}{l}\text { Desviación } \\
\text { estándar }\end{array}$ & 0.009 & 0.02 & 0.04 & 17.55 & 15.07 & 12.76 \\
\hline Observaciones & 603 & 650 & 639 & 650 & 650 & 650 \\
\hline \multicolumn{7}{|l|}{ Muestra XIV } \\
\hline Media & 0.01 & 0.01 & 0.01 & 57.56 & 61.69 & 62.56 \\
\hline Máximo & 0.05 & 0.19 & 0.29 & 91.67 & 88.64 & 86.22 \\
\hline Mínimo & 0.0008 & 0.0002 & 0.00007 & 24.07 & 30.30 & 29.49 \\
\hline $\begin{array}{l}\text { Desviación } \\
\text { estándar }\end{array}$ & 0.006 & 0.02 & 0.02 & 16.87 & 14.54 & 12.54 \\
\hline Observaciones & 615 & 650 & 640 & 640 & 640 & 640 \\
\hline
\end{tabular}

Fuente: Elaboración propia

Tabla 1c. Resultados de las muestras $V I, V I I, V I I I, X, X I, X I I$ y $X I V$ con el método de efectos aleatorios de una vía (período).

\begin{tabular}{|c|c|c|c|c|c|c|c|}
\hline & $\boldsymbol{V I}$ & $\boldsymbol{V I I}$ & $\boldsymbol{V I I I}$ & $\boldsymbol{X}$ & $\boldsymbol{X I}$ & $\boldsymbol{X I I}$ & $\boldsymbol{X} \boldsymbol{V}$ \\
\hline $\boldsymbol{c}$ & $0.0153^{* * *}$ & $0.0158^{* * *}$ & $0.0151^{* * *}$ & $0.0087^{* * *}$ & $0.0053^{* * *}$ & $0.0061^{* * *}$ & $0.0074^{* * *}$ \\
\hline$g c c$ & -0.0087 & 0.0161 & $0.0432^{*}$ & -0.0015 & -0.0093 & 0.0012 & 0.0125 \\
\hline$g d c$ & -0.0020 & 0.0174 & 0.0071 & $-0.0302^{* *}$ & -0.0066 & -0.0056 & -0.0193 \\
\hline $\boldsymbol{i s}$ & $0.0004^{* * *}$ & $0.0002^{* * *}$ & $0.0002^{* * *}$ & $0.0002^{* * *}$ & 0.0001 & -0.000007 & $0.0002^{* * *}$ \\
\hline ich & $-0.0004^{* * *}$ & $-0.0004^{* * *}$ & $-0.0006^{* * *}$ & $-0.0006^{* * *}$ & $-0.0002^{* *}$ & -0.00004 & $-0.0002^{* *}$ \\
\hline irg & 0.00006 & 0.0002 & $0.0003^{* * *}$ & $0.0005^{* * *}$ & $0.0003^{* * *}$ & $0.0001^{*}$ & $0.0001^{*}$ \\
\hline $\begin{array}{c}\text { Estadísticas } \\
\text { R-cuadrado }\end{array}$ & 0.04 & 0.04 & 0.04 & 0.06 & 0.05 & 0.03 & 0.04 \\
\hline $\begin{array}{c}\text { Estadístico Durbin } \\
\text { Watson }\end{array}$ & 1.02 & 0.95 & .93 & .83 & 1.27 & 1.28 & 0.93 \\
\hline $\begin{array}{c}\text { Prueba Hausman } \\
\text { de efectos } \\
\text { aleatorios } \\
\text { correlacionados } \\
\text { (período) }\end{array}$ & 0.19 & 0.12 & 0.57 & 0.75 & 0.27 & 0.27 & 0.14 \\
\hline \begin{tabular}{c} 
Observaciones \\
\hline
\end{tabular} & 620 & 572 & 539 & 710 & 649 & 698 & 595 \\
\hline
\end{tabular}

Fuente: Elaboración propia 